ACCEPTED MANUSCRIPT

\title{
Influence of nanoholes array geometrical parameters on magnetic properties of Dy-Fe antidot thin films
}

To cite this article before publication: Mohamed Salaheldeen Mohamed Hassan et al 2019 Nanotechnology in press https://doi.org/10.1088/1361$\underline{6528 / a b 36 c c}$

\author{
Manuscript version: Accepted Manuscript \\ Accepted Manuscript is "the version of the article accepted for publication including all changes made as a result of the peer review process, \\ and which may also include the addition to the article by IOP Publishing of a header, an article ID, a cover sheet and/or an 'Accepted \\ Manuscript' watermark, but excluding any other editing, typesetting or other changes made by IOP Publishing and/or its licensors" \\ This Accepted Manuscript is @ 2019 IOP Publishing Ltd.
}

During the embargo period (the 12 month period from the publication of the Version of Record of this article), the Accepted Manuscript is fully protected by copyright and cannot be reused or reposted elsewhere.

As the Version of Record of this article is going to be / has been published on a subscription basis, this Accepted Manuscript is available for reuse under a CC BY-NC-ND 3.0 licence after the 12 month embargo period.

After the embargo period, everyone is permitted to use copy and redistribute this article for non-commercial purposes only, provided that they adhere to all the terms of the licence https://creativecommons.org/licences/by-nc-nd/3.0

Although reasonable endeavours have been taken to obtain all necessary permissions from third parties to include their copyrighted content within this article, their full citation and copyright line may not be present in this Accepted Manuscript version. Before using any content from this article, please refer to the Version of Record on IOPscience once published for full citation and copyright details, as permissions will likely be required. All third party content is fully copyright protected, unless specifically stated otherwise in the figure caption in the Version of Record.

View the article online for updates and enhancements. 


\title{
Influence of nanoholes array geometrical parameters on magnetic properties of Dy-Fe antidot thin films
}

\author{
M. Salaheldeen ${ }^{* 1,2}$, V. Vega ${ }^{3}$, R. Caballero-Flores ${ }^{2}$, V.M. Prida ${ }^{2}$, A. Fernández \\ 1) Physics Department, Faculty of Science, Sohag University, 82524 \\ Sohag, Egypt \\ 2) Depto. Física, Universidad de Oviedo, C/ Federico García Lorca 18, \\ 33007 Oviedo, Asturias, Spain \\ 3) Lab. Membranas Nanoporosas, Servicios Científico-Téenicos, \\ Universidad de Oviedo, Campus El Cristo s/n 33006 Oviedo, Asturias, \\ Spain. \\ *e-mail: m.salaheldin@science.sohag.edu.eg
}

\begin{abstract}
.
Nanoscale artificially engineered spintronic materials could be used to enlarge the storage density of magnetic recording media. For this purpose, magnetic nanostructures such as antidot arrays exhibiting high uniaxial magnetic anisotropy are new contestants in the field of ultrahigh density magnetic data storage devices. In this context, we focus on the synthesis of nanostructured magnetic materials consisting of Dy-Fe alloyed antidot thin films, deposited onto the surface of nanoporous alumina membranes served as patterned templates. Noticeable variations of in the in-plane magnetic anisotropy have been observed by modifying the layer thickness at both microscopic and macroscopic scales. The microscopic magnetic properties have been locally studied by Nano-MOKE magnetometry. For thinner antidot samples with 15, 20 and $25 \mathrm{~nm}$ in thickness, a tri-axial inplane magnetic anisotropy has been detected. Meanwhile, for thicker antidot samples (40-60 $\mathrm{nm}$ of layer thickness), an in-plane uniaxial magnetic anisotropy has been noted. We attribute these changes in the magnetic anisotropy to the strong correlation between the edge-to-edge distance among adjacent nanoholes, $W$, and the local magnetic anisotropy of antidot samples. The effective magnetic anisotropy exhibits an unexpected crossover from the in-plane to out-of-plane direction due to the increasing of the effective perpendicular magnetic anisotropy with varying the layer thickness of antidot thin films. Therefore, we detected a critical layer thickness, $t=25 \mathrm{~nm}$ for the Dy-Fe alloy antidot arrays, at which the appearance of the perpendicular magnetization is observed. Furthermore, an enhancement in the Curie temperature of the antidot arrays compared to the continuous thin films has been obtained. We attribute these effects to the complex magnetization reversal processes and the high thermal stability of the hexagonal structure of antidot arrays. These findings can be
\end{abstract}


Influence of nanoholes array geometrical parameters

of high interest for the development of novel magnetic sensors and for thermo-magnetic recording patterned media based on template-assisted deposition techniques.

Keywords: nanoporous alumina templates; antidot arrays; Kerr effect; perpendicular magnetic anisotropy; Curie temperature; spintronics. 
Influence of nanoholes array geometrical parameters

\section{Introduction}

Highly spatially ordered nanohole arrays embedded in magnetic thin films, called antidot arrays, have been investigated recently for various materials. In the dynamic regime, these antidots can act as magnon crystals providing an improved control of spin waves propagation $[1,2]$. On the other hand, antidot arrays have been suggested as noyel prototypes of bit-patterned storage media that can exceed the superparamagnetic limit, as there are not isolated islands due to they are usually found in isolated magnetic dots [3]. Therefore, thin films of antidot arrays are being intensely studied as candidates for many applications such as in high-density magnetic data storage, [4,5] magnetically active plasmonics, [6] microwave devices, [7] artificial spin ice, magneto-optic perpendicular recording patterned media [8] and magnetic biosensor applications [9]. Actually, the nanoholes can act as pinning centres for the magnetic domain walls, DW, and hinder their propagation through magnetic thin film. They also induce a local shape anisotropy that tends to align the magnetization parallel to the hole's edges [10]. This effect is widely used to tailor the magnetic properties of these thin films such as their magnetic anisotropy, $[8,11]$ coercive field, remanence and switching mode of the magnetization reversal process [12]. This can be done through the control of the geometrical parameters of the antidot arrays, such as the nanometric size and shape of the holes, interdistance between them, periodic spatial ordering of their geometrical arrangement and the film thickness [13-18].

Both, ferrimagnetic amorphous and crystalline rare earth-transition metal (RETM) compounds and their alloys, in either the bulk material or thin films, have attracted great attention in the past as a potential medium for thermo-magnetic recording and applications in modern ultrafast storage [19-23]. The importance nowadays of amorphous materials (RE-TM), is found mainly in the research field of partial exchange 
Influence of nanoholes array geometrical parameters

heterostructures [23], giving place to the research fields of giant exchange bias [24], alloptical switching, magnetic data storage applications and spintronics due to the fact that they meet all the requirements for an effective magneto-optical material [21-23. 25]. The materials most commonly used for these applications are based on RE-TM alloys, $(\mathrm{RE}=$ $\mathrm{Tb}, \mathrm{Gd}, \mathrm{Dy})$ and $(\mathrm{TM}=\mathrm{Fe}, \mathrm{Co}, \mathrm{Ni})[26]$. Magnetic studies of amorphous $\mathrm{RE}_{\mathrm{x}}-\mathrm{Fe}_{(100-\mathrm{x})}$ thin films indicate that, for the heavier rare-earth metals, these films show a ferromagnetic behaviour [24,27]. Recently, RE-TM nanostructures based on antidots have shown a strong potential for energy-assisted recording on nanometre-scale magnetic media, magneto-optic perpendicular recording patterned media based on template-assisted deposition techniques and ultrafast spintronic technology $[8,28-30]$

In this work, we pay special attention to the basic magnetic properties such as the magnetic anisotropy, Curie temperature, effective magnetic anisotropy energy, remanence and coercivity angular dependence, of Dy-Fe alloy antidot arrays by studying the effect of the geometrical parameters as they are, shape and size of nanohole, holes spatial ordering and antidot film thickness, on their magnetic properties. In addition, the influence of hexagonal ordering symmetry of nanoholes arrangement on the magnetothermal behaviour as compared to the continuous thin film samples has been investigated.

\section{Experimental procedure}

\subsection{Fabrication of Dy-Fe alloyed antidot thin films}

Two series of hexagonally ordered (HAD) and disordered (DAD) antidot arrays of Dy-Fe alloy thin films having an interhole distance $\boldsymbol{D}_{\text {int }}=105 \mathrm{~nm}$, hole diameter, $\boldsymbol{d}=$ $80 \pm 3 \mathrm{~nm}$ and film thickness varying between $15 \mathrm{~nm} \leq t \leq 60 \mathrm{~nm}$ (with increasing steps of each $5 \mathrm{~nm}$ ), were grown by high-vacuum thermal evaporation technique. The continuous 
Influence of nanoholes array geometrical parameters

Dy-Fe thin films, CTF, were deposited also on a glass substrate, with the same thicknesses than for the antidots samples, to compare the obtained results.

In order to prepare hexagonally ordered antidot arrays, nanoporous alumina membranes were produced through the two-step anodization process [31,32]. High purity Al foils (99.999\%, Goodfellow, Huntingdon, UK), with $0.5 \mathrm{~mm}$ of thickness, were cleaned by sonication in ethanol and isopropyl alcohols, then they were electropolished at $20 \mathrm{~V}$ in perchloric acid and ethanol solution $\left(1: 3 \mathrm{vol} ., 5^{\circ} \mathrm{C}\right)$ for $5 \mathrm{~min}$. The polished $\mathrm{Al}$ foils were employed as starting specimen for the anodic synthesis of nanoporous alumina templates. The two steps electrochemical anodization procedure was done in $0.3 \mathrm{M}$ oxalic acid, at a temperature ranging between $1-3^{\circ} \mathrm{C}$ and under a potentiostatic applied voltage of $40 \mathrm{~V}$, measured versus a Pt counter electrode. To obtain the highly ordered nanoporous alumina templates, the samples were immersed in $0.2 \mathrm{M} \mathrm{CrO}_{3}$ and $0.6 \mathrm{M} \mathrm{H}_{3} \mathrm{PO}_{4}$ aqueous solution. This selective chemical etching step leads to the selective removal of the first grown anodic alumina layer, which contained randomly disordered nanopores at its top surface as show in figure $1 \mathrm{a}$. For the alumina templates with hexagonal lattice, the second anodization step has been performed during $5 \mathrm{~h}$., allowing the nanopores growth by following a highly self-ordered hexagonal symmetry. In contrast, the disordered nanoporous alumina samples were only submitted to a single anodization step, and therefore the sample surface remains with randomly distributed nanopores grown during the first stages of the anodization procedure (figure 1a).

Finally, nanoporous alumina samples were chemically etched in 5 wt. \% orthophosphoric acid at $30{ }^{\circ} \mathrm{C}$, for 35 minutes. This procedure allowed us to obtain nanoporous alumina templates with pore diameter of $80 \pm 3 \mathrm{~nm}$ and keeping the interpore distance constant, $D_{\text {int }}$, at $105 \pm 3 \mathrm{~nm}$, as show in figure $1 \mathrm{~b}$. Finally, the controlled deposition of the metallic Dy-Fe alloy film formed by highly pure metal pieces of Fe 
Influence of nanoholes array geometrical parameters

(Goodfellow Limited, England, 99.9\% purity) and Dy (Ventron GMBH, Germany, 99.99\% purity) was completed by a high vacuum thermal evaporation technique using an E306A thermal vacuum coating unit (Edwards, Crawley, UK), with an ultimate vacuum better than $5.2 \times 10^{-7}$ mbar, having a diffusion pump backed by rotary pumping together with a liquid nitrogen trap [33]. The pure element metal pieces were placed inside water cooled copper crucible and have been heated by magnetically focused electron beam, 3.1 $\mathrm{kV}$ for Fe (crucible1) and $4.5 \mathrm{kV}$ for Dy (crucible2) and electric energy $2.7 \mathrm{~kW}(\mathrm{Fe})$ and $2.5 \mathrm{~kW}$ for (Dy). The evaporated target metals were deposited on the top-surface of both, the hexagonally ordered and randomly disordered nanoporous alumina membranes, which play the role as templates to obtain the thin films of antidot arrays [13,34]. The control of the film thickness was achieved by using two independent quartz crystal controllers that monitored simultaneously the deposition rates of each evaporation source. The source to substrate distances were maintained constant at about $18 \mathrm{~cm}$. The deposition rate of the Dy-Fe alloy was around $0.1-0.15 \mathrm{~nm} \mathrm{~s}^{-1}$. The experimental details about the deposition conditions have been already reported elsewhere $[8,35]$.

\subsection{Morphological characterization}

\subsubsection{Scanning Electron Microscopy analysis}

The morphological characterization of samples was performed by means of Scanning Electron Microscopy technique (SEM) (JSM 5600, JEOL, Akishima, Tokyo, Japan). Figure 1(c to h) shows SEM images for sample surfaces of Dy-Fe antidot arrays with same hexagonal symmetry and starting values of lattice parameters $\left(d, D_{\text {int }}\right)$, but varying the thickness layer. We should point out that as the thickness of the film increases, the apparent diameter of the nanoholes reduces due to the deposition of magnetic material in the top of the hole until it totally collapses for layer thickness above $60 \mathrm{~nm}$ (figure $1 \mathrm{~h}$ ). 

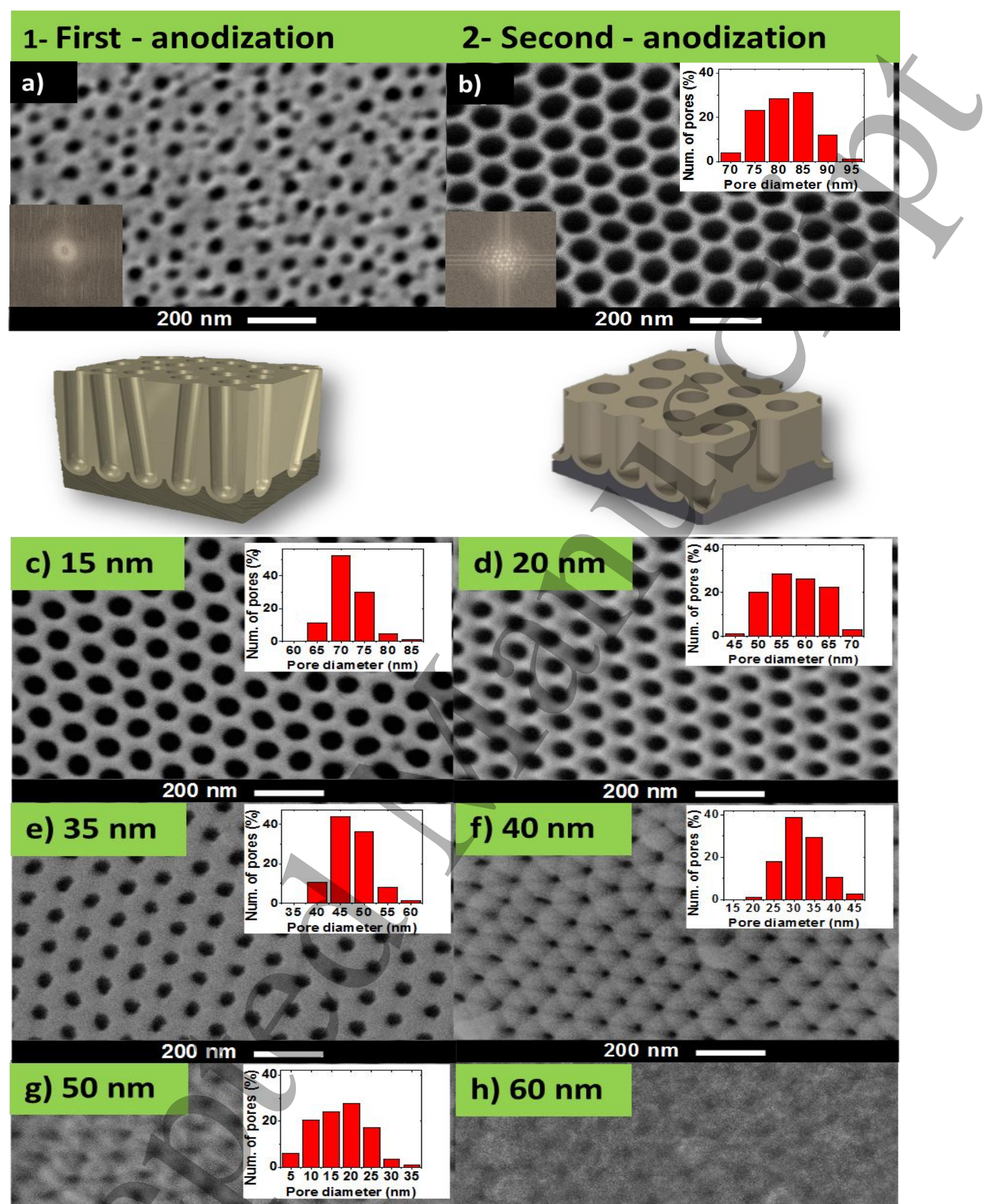

$200 \mathrm{~nm}$

Figure 1: a) and b) show SEM images for the first anodization the second anodization to obtain the showing only a halo that corresponds to the starting alumina membrane with disordered nanopores and $b$ ) illustrating the hexagonal ordering of nanopores). c) to h) SEM images of series from Dy-Fe antidot arrays with layer thickness varying from $15 \mathrm{~nm}$ up to $60 \mathrm{~nm}$. 


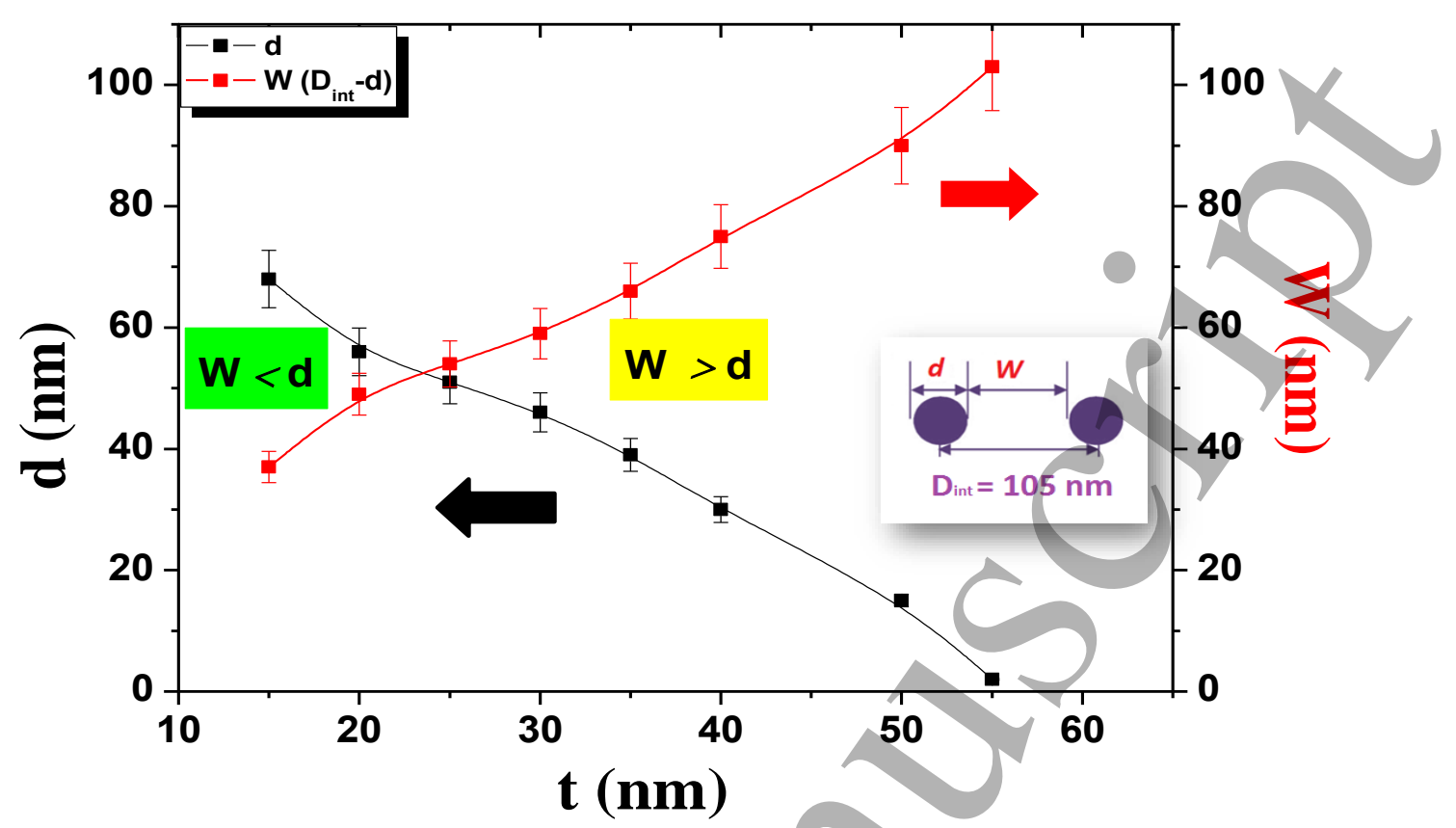

Figure 2: Dependence of the apparent nanoholes diameter, $\boldsymbol{d}$, and the edge-to-edge antidot distance, $W$, of the Dy-Fe antidot arrays with the film thickness grown between $15 \mathrm{~nm}$ and $55 \mathrm{~nm}$.

Figure 2 shows the correlation between the apparent nanoholes diameter, $d$, and the edge-to-edge antidot distance, $W=\left(D_{\text {int }}-d\right)$, with the antidot layer thickness, $t$, of the Dy-Fe alloy antidots. This change in the apparent nanohole diameter is due to the deposition of magnetic material in the upper part of the wall of the pore. From figure 2 , it can be distinguished two regimes depending on the value of the edge-to-edge antidot distance. First one corresponds to the case when $W$ is smaller than the antidot hole diameter i.e. (thinner samples), and later when $W$ is bigger than the antidot diameter i.e. (thicker films).

Microscopically, the nanoporous alumina templates show a well-defined hexagonal lattice which geometrical properties have been characterized by SEM measurements, as shown in Figure 1. The hexagonal geometry of the antidot arrays allows distinguishing some characteristic main directions in the samples surface. Firstly, 
Influence of nanoholes array geometrical parameters

choosing any particular antidot as the origin, the direction from the origin to the nearest neighbouring antidot, $(\mathrm{NN})$, and secondly, the direction from the antidot of origin to the second nearest neighbour, $(\mathrm{NNN})$, as shown in Figure 3 a. These directions alternate every $30^{\circ}$ all around, with symmetry of $60^{\circ}$. Thus, it can be detected an angular magnetic anisotropy dependence in the plane of the antidot arrays films, related to these two main directions, as it will be discussed in section 3.2.1, and also reported elsewhere, [36].
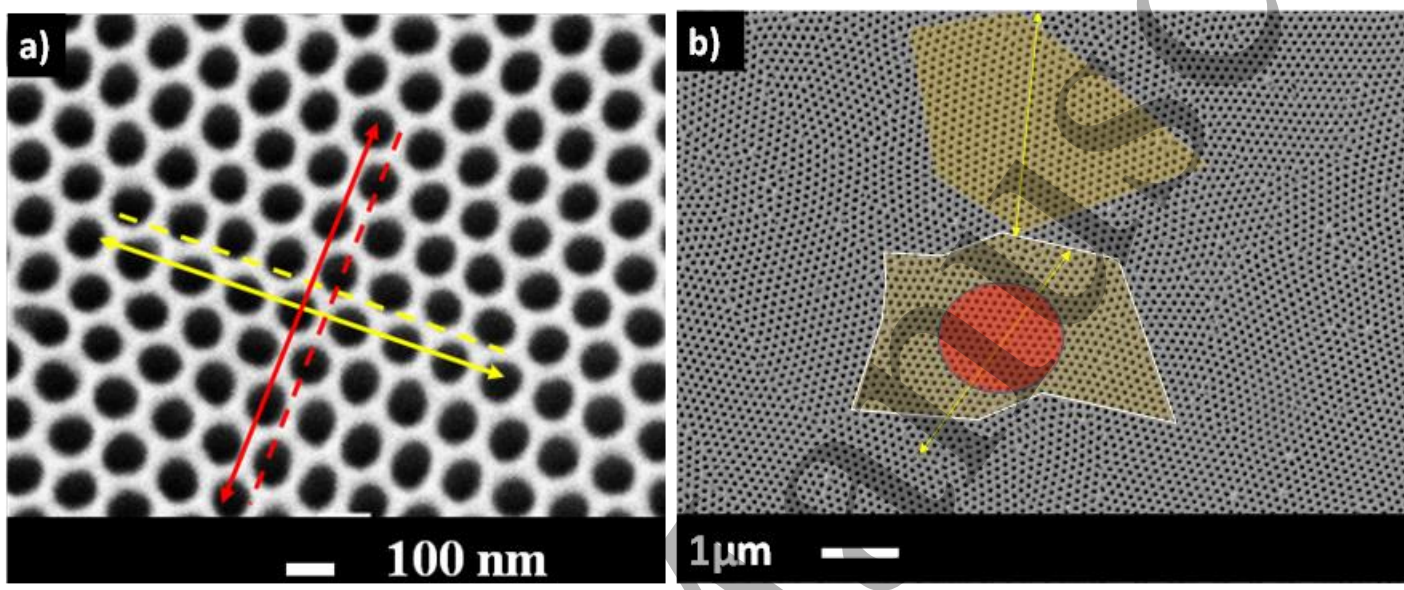

Figure 3: a) SEM images of antidot substrate illustrating the near neighbour, NN, (double arrow continuous yellow line) and its parallel line (dashed yellow line) along the edges of nanoholes and a next near neighbour, NNN, direction (double arrow continuous red line) and its parallel line (dashed red line). b) SEM image of the surface of an antidots thin film with low magnification.

Macroscopically, at low magnification the nanoporous alumina templates present a hexagonal order of circular holes, divided into geometric hexagonal domains separated by dislocation-like boundaries as shown in figure $3 \mathrm{~b}$. The typical size of the hexagonally ordered pore domains is in the range of 4-6 micrometers, as highlighted in different colors in Figure 3b. In addition, this figure represents two NN directions, one in each domain, showing the different orientation of the hexagonal ordering in them. Both $\mathrm{NN}$ directions differ in $25^{\circ}$, while if they belonged to the same hexagonal order should match or differ in $60^{\circ}$ or $120^{\circ}$. When comparing the lithographed antidot arrays [15] with the ones obtained using anodic alumina membranes, the latter displays smaller holes sizes and 
Influence of nanoholes array geometrical parameters

lattice parameters. However, the main drawback relies on the presence of geometric hexagonal domains, while lithographed arrays are mono-domain.

\subsubsection{Chemical composition}

The chemical composition of Dy-Fe antidots thin films and their corresponding continuous thin film are initially calculated from the measurements provided by the controls of crystal quartz balance at the end of the film deposition, used to calculate the ratio $\mathrm{RE} / \mathrm{TM}$ in the alloy. To confirm the nominal composition of the alloy, we perform a subsequent analysis by energy dispersive X-ray spectroscopy (EDX). The analysis of the spectra of three of these films shows a $\mathrm{Dy}_{13} \mathrm{Fe}_{87}$ averaged composition as summarized in the table 1 and the measurements agree with which obtained by crystal quartz balance.

Table 1: Atomic percentage of Fe and Dy elemental composition in Dy-Fe thin film.

\begin{tabular}{|l|l|l|}
\hline EDX Spectrum & \multicolumn{1}{|c|}{ Fe $($ at. \%) } & Dy (at.\%) \\
\hline Spectrum1 & 86.9 & 13.2 \\
\hline Spectrum2 & 87.7 & 12.3 \\
\hline Spectrum3 & 87.2 & 12.8 \\
\hline A verage & $\mathbf{8 7 . 3}$ & $\mathbf{1 2 . 7}$ \\
\hline
\end{tabular}

\subsection{Microstructural characterization by Transmission Electron Microscopy} analysis.

The microstructure of the samples has been studied by high-resolution transmission electron microscopy (HR-TEM) (JEM 2100, JEOL, Akishima, Tokyo, Japan) operating at $200 \mathrm{kV}$, which was employed to obtain high magnification images of the antidots thin films. For that purpose, the nanoporous alumina membrane that acts as the template for the fabrication of the antidot arrays was previously and selectively 
Influence of nanoholes array geometrical parameters

dissolved in a $0.5 \mathrm{M} \mathrm{NaOH}$ solution, thus releasing the free-standing flakes of the nanostructured thin film, which were then washed with distilled water and ethanol, deposited into conventional transmission electron microscopy (TEM) copper grid sample holders and dried in air. Selected area electron diffraction (SAED) spectrum was performed to study the microstructure of antidot arrays samples.

The TEM micrograph of the Dy-Fe antidots thin film obtained after being released from the nanoporous alumina membrane is displayed in Figure 4, which demonstrates that the nanometric holes successfully replicated the structure of the highly hexagonal ordered nanoporous alumina template, in agreement with the findings revealed by the SEM images. Also, the SAED spectrum, shown as the inset in Figure 4a, indicates the amorphous structure of the Dy-Fe alloy, evidenced by the presence of diffused rings and the absence of clear spots in the electron diffraction spectrum.
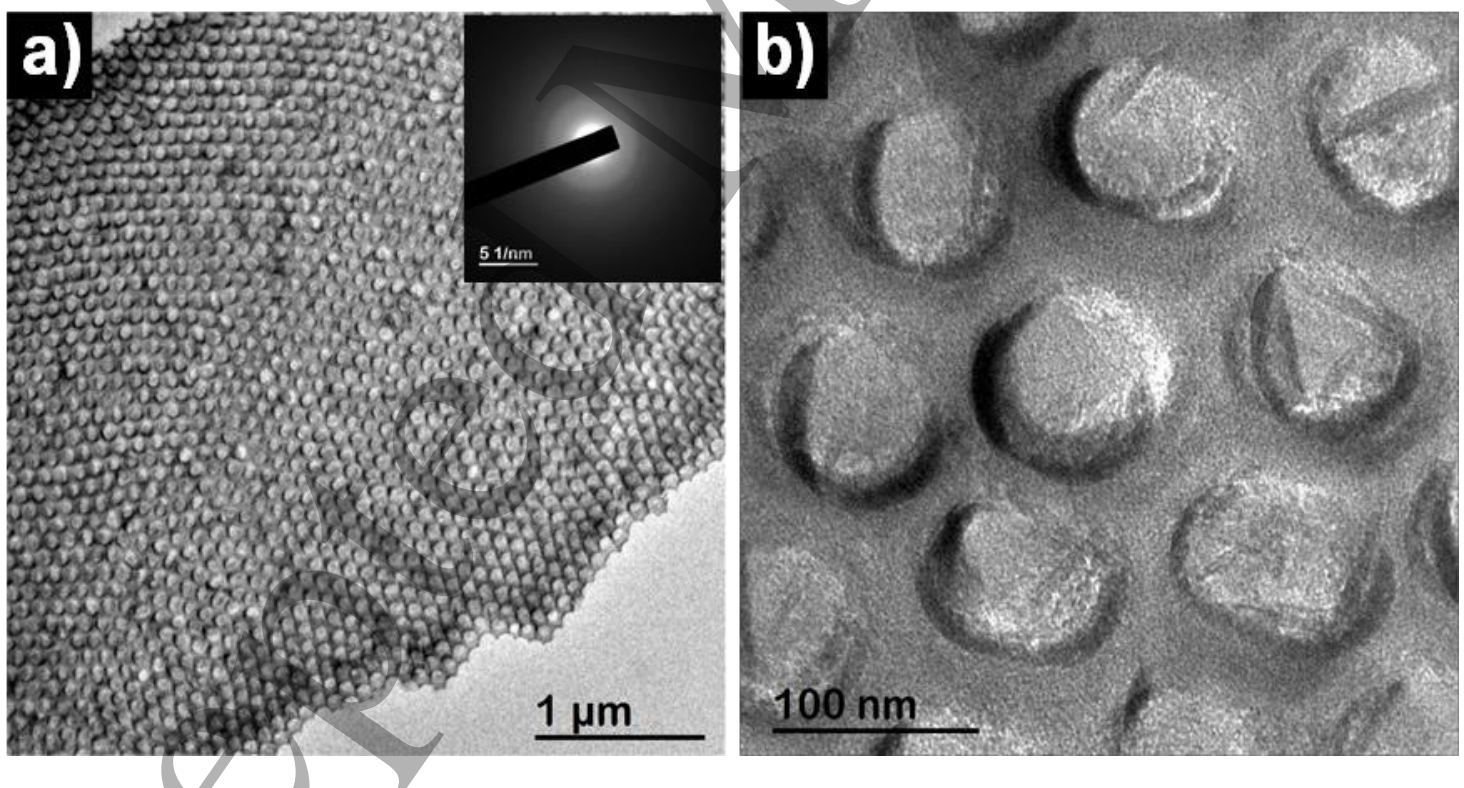

Figure 4: HR-TEM images of Fe-Dy antidot arrays with $15 \mathrm{~nm}$ in layer thickness taken at low (a) and high (b) magnification. The inset in (a) shows the SAED spectrum.

\subsection{Characterization of magnetic properties}


Influence of nanoholes array geometrical parameters

Magnetic properties of Dy-Fe alloy antidot arrays and their corresponding CTF have been determined from the hysteresis loops measured at room temperature on samples surface by the magneto-optical Kerr effect (MOKE), with applied magnetic field of up to $\pm 5 \mathrm{kOe}$, using the NanoMOKE-3 magnetometer, (Durham Magneto Optics Ltd.). In addition, we have also measured the bulk hysteresis loops of the samples by using a vibrating sample magnetometer, (VSM-QD-Versalab) with applied magnetic fields up to $\pm 30 \mathrm{kOe}$. In both cases, the magnetic field is applied in the plane of the film, INP, and perpendicular to the film plane (out of plane), OOP, directions. NanoMOKE-3 magnetometer allows obtaining local measurements of the magnetization of the sample by employing a focused laser beam covering an area smaller than 2 microns of diameter, as show in figure $3 \mathrm{~b}$. Alternatively, a defocusing lens can be employed in order to widen the laser beam, covering an area of about $0.5 \mathrm{~mm}$ radius, thus widening the area of measurement. Therefore, it is possible to obtain information from the magnetization of the sample at the microscopic or macroscopic level.

First, we choose the local, microscopic, settings for NanoMOKE-3 magnetometer. The magneto-optical measurements were also performed for different orientations of the INP applied field. Hysteresis loops were obtained for both types of samples, CTF and antidot arrays films. The angular dependence of the magnetic properties was studied by rotating the samples every 5 degrees in the plane of the sample. To avoid the translational movement due to the sample rotation, we firstly localized a specific feature of the sample surface by using the scanning laser microscopy option of Nano-MOKE. Then we start the measurement at a well-controlled position located at fixed distance, which is about $10 \mu \mathrm{m}$ from this point. Therefore, we correct the sample translation displacement that happens because of stage rotation for every measurement angle by using the same procedure. 
Influence of nanoholes array geometrical parameters

\section{Magnetic behaviour of Dy-Fe continuous thin films and antidots arrays.}

\subsection{Magnetic properties of continuous thin films}

The INP MOKE hysteresis loop of selected Dy-Fe alloy CTF with thickness ranging between $15 \mathrm{~nm}$ and $60 \mathrm{~nm}$ are shown in figure 5 (a, c and e). A clear in-plane uniaxial anisotropy has been observed for all CTF samples, where the angular dependence of the in-plane coercivity, $H_{C}(\theta)$, shows a two-fold symmetry. Actually, amorphous RETM alloys exhibit an anisotropic microstructure when prepared by physical vapor deposition onto room-temperature substrate that is called as columnar structure $[37,38]$. Usually, this columnar micromagnetic structure induces an in-plane uniaxial magnetic anisotropy in the thin films [38]. Therefore, we called this anisotropy as "deposition intrinsic anisotropy" of CTF, which is induced during the film deposition. Its values range from $3 \times 10^{6}$ to $8 \times 10^{6}\left(\mathrm{erg} / \mathrm{cm}^{3}\right)$. Also, this in-plane intrinsic anisotropy is found for all continuous thin films with thickness ranging from 15 to $60 \mathrm{~nm}$ and it follows a two-fold order symmetry. It has been observed that increasing the layer thickness of Dy-Fe CTF the coercivity multiplied with the film thickness, $H_{C} \times t$, increase from $0.75 \mathrm{kOe} \mathrm{nm} \mathrm{up} \mathrm{to}$ $22 \mathrm{kOe} \mathrm{nm}$ for samples with layer thickness of $15 \mathrm{~nm}$ and $60 \mathrm{~nm}$, respectively. Actually, the parameter $H_{C} \times t$ is related to the local domain wall pinning force within the alloy and this observed change with film thickness may well indicate the onset of associated changes in the film morphology [39]. 
Influence of nanoholes array geometrical parameters
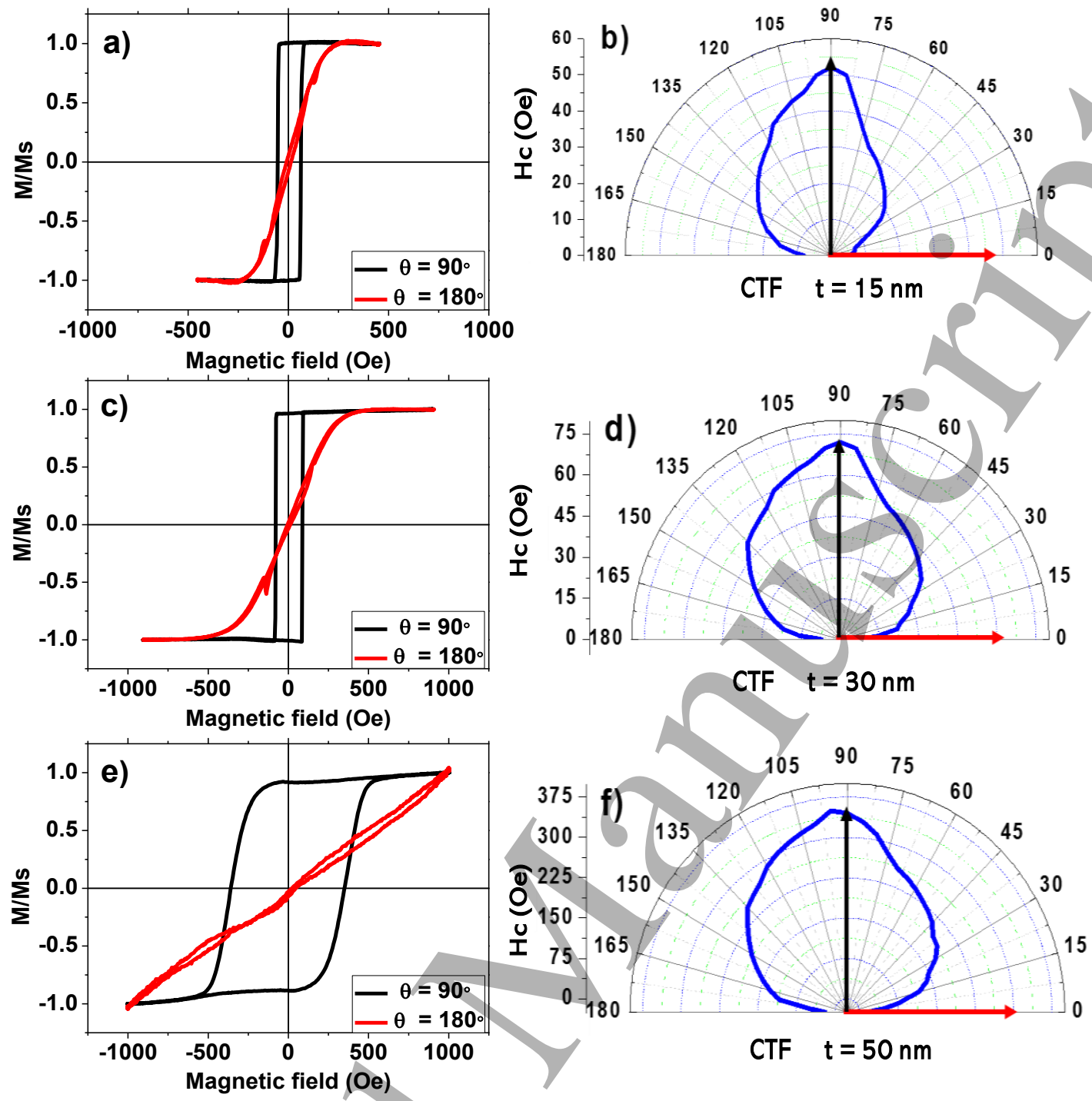

Figure 5: In-plane hysteresis loops of Dy-Fe alloy continuous thin film and related INP angular coercivity dependence for samples with different thickness: a) and b) $15 \mathrm{~nm}$, c) and d) $30 \mathrm{~nm}$ and e) and f) $50 \mathrm{~nm}$, respectively.

\subsection{Antidot arrays thin films}

\subsubsection{Antidots with hexagonal symmetry}

The in-plane surface magnetic properties of Dy-Fe antidot arrays samples with different layer thickness are plotted in the Figure 6. Several differences in the in-plane magnetic properties of Dy-Fe antidots have been found comparing to their corresponding CTF. Firstly; the in-plane hysteresis loop loses its squareness and becomes wider. Also, 
Influence of nanoholes array geometrical parameters

the INP loops show multi-step magnetization behaviour which indicates a strong pinning effect and complex magnetization reversal process as shown in figure $6(\mathrm{a}, \mathrm{c})$. This multistepped magnetic behaviour indicates that there is a contribution of the out of plane component in the magnetization reversal mechanism [40,41]. Meanwhile, the hysteresis loops of the thicker samples exhibit a single magnetic behaviour as shown in figure $6(\mathrm{e}$, and g). Secondly, a sharp increase in the $H_{C}$ for the INP and OOP directions is observed for the antidot thin film comparing to the CTF. The maximum $H_{C}$ value of 848 Oe has been obtained for antidot samples with layer thickness of $15 \mathrm{~nm}$. This value of coercivity is approximately 17 times larger than the corresponding one for CTF. These differences between CTF and HAD can be explained because of a change in the magnetization reversal mechanism from domain wall movement to super domain wall pinning, as reported in [42], which induced by the nano-holes arrangement, respectively [15, 16, 32].

For a best understanding of the magnetic properties of Dy-Fe antidot arrays, we study the angular dependence of the in-plane coercivity and remanence that can be extracted from the INP hysteresis curves. Figure $6(\mathrm{~b}, \mathrm{~d}$, and $\mathrm{f})$ represents the angular dependence of $H_{C}(\theta)$ for antidots arrays thin films with 15, 20,30 and $50 \mathrm{~nm}$ in thickness. We observe for the sample with lower film thicknesses (15 and $20 \mathrm{~nm})$ a six-fold order symmetry of $H_{C}(\theta)$, where the easy anisotropy axis, E. A., (highest values of $H_{C}$ ) repeated every $60^{\circ}$ and the difference between the E.A. and hard anisotropy axis, H.A., is $30^{\circ}$ as it has been reported also in the case of lithographed antidot arrays [15]. For antidot samples with layer thickness of $30 \mathrm{~nm}$ and $35 \mathrm{~nm}$ they exhibit an out-of-plane anisotropy, but when we restrict the study to the in-plane magnetization hysteresis loops a four-fold symmetry of the $H_{C}(\theta)$ has been detected, where the E.A repeated every $90^{\circ}$ and the difference between the E.A and the H.A is $45^{\circ}$ as show in (figure $6 \mathrm{f}$ ). Finally, for thicker antidot samples $(50 \mathrm{~nm})$, two-fold symmetry of $H_{C}(\theta)$ was observed, where the E. A. 
Influence of nanoholes array geometrical parameters

repeated every $180^{\circ}$ and the difference between the E. A. and the H.A. is $90^{\circ}$ i.e. the same behaviour of CTF as show in (figure $6 \mathrm{~h}$ ) and figure 5. Consequently, we can deduce that there is a critical geometric parameter where the six-fold order symmetry for the Dy-Fe antidot thin film can be affected. For HAD samples which size of lattice parameters are below this critical value of the geometrical parameter, the effective INP local magnetic anisotropy, induced by holes, is dominating and causes the local six-fold order anisotropy for the antidot arrays with hexagonal symmetry. Whereas, for antidot samples with $W>$ $d$, i.e. higher than the critical point of the geometrical parameter, the effective in-plane local magnetic anisotropy becomes weaker because of the increasing contribution of intrinsic magnetic anisotropy of the continuous thin film area between the adjacent holes. Therefore, different magnetic anisotropy contribution and symmetry appeared. It is known, that the coercivity of the antidot arrays thin film is determined as a balance between the intrinsic magnetic anisotropy of thin film and the shape anisotropy induced by the arrays of holes $[35,36,38]$.

If we now choose the macroscopic, or non-local configuration for the laser beam of the NanoMOKE-3 magnetometer, the area of sample surface covered by the laser beam has a diameter of the order of $0.5 \mathrm{~mm}$. Under these measurement conditions, the MOKE signal is not proportional to the magnetization of only one single domain of antidots with hexagonal ordering, but it is the result of the sum of the MOKE response in multitude of hexagonally ordered domains of antidots. 
Influence of nanoholes array geometrical parameters
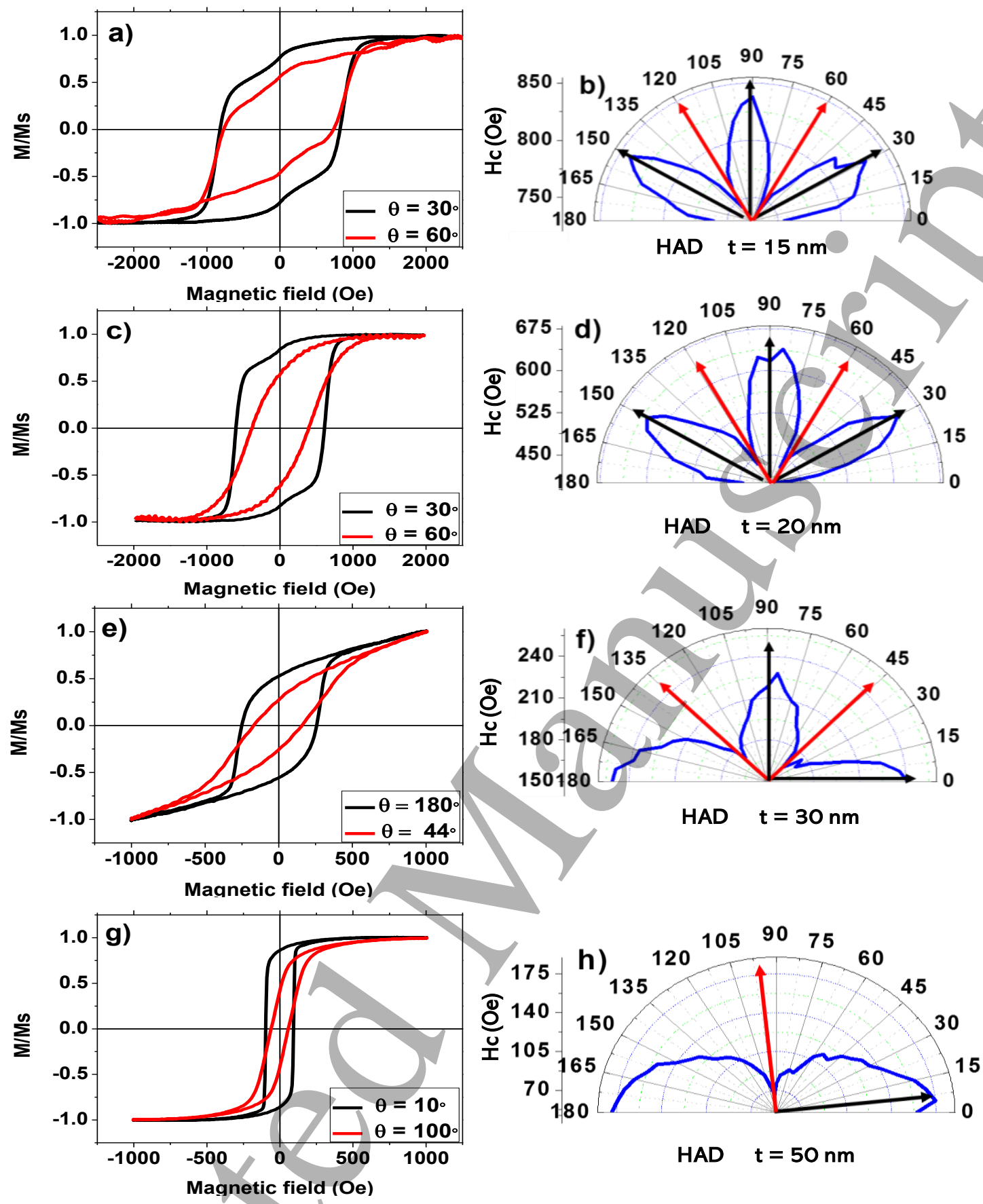

Figure 6: The in-plane hysteresis loops of Dy-Fe alloy antidot thin films with different thicknesses and their related INP angular coercivity dependence for: a) and b) $15 \mathrm{~nm}$, c) and d) $20 \mathrm{~nm}$ and e) and f) $50 \mathrm{~nm}$.

Since the orientation of the hexagonal ordering of antidots will change from one domain to another, the six-fold order symmetry observed in the magnetic properties, i.e. angular dependence of $H_{C}$ or $m_{r}$, of a single domain of hexagonal order is not found when using the macroscopic configuration of the laser beam for studying bigger sample surface 


\section{Influence of nanoholes array geometrical parameters}

areas, as shown in Figure $7(\mathrm{a}, \mathrm{b})$. These results fit better within a two-fold order symmetry, likely due to an in-plane uniaxial anisotropy.
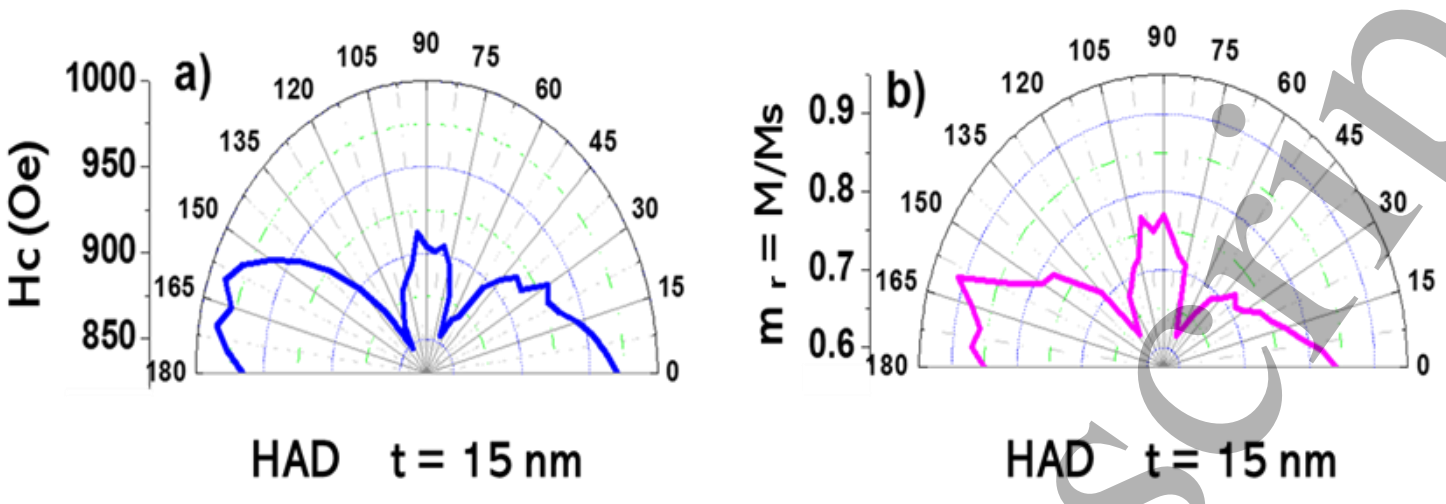

Figure 7: In-plane angular dependence of, a) $H c$ and b) $m_{r}$, for an antidot array thin film with $15 \mathrm{~nm}$ in layer thickness, when using the macroscopic configuration of the laser beam of NanoMOKE-3 magnetometer.

\subsubsection{Antidot arrays with disordered nanoholes arrangement.}

In the case of a DAD array with no hexagonal ordering of nanoholes and therefore no geometric configuration of hexagonal domains of antidot arrays, the local six-fold order anisotropy is lost. As previously mentioned in the experimental section, the nanopores arrangement obtained at the sample surface after the first anodization process is randomly disordered. The dislocations are inexistent in this kind of nanoholes arrays, and the sample can be compared to an amorphous packing of antidots with a broad distribution of holes diameter. Figure $8(\mathrm{a}, \mathrm{c})$ represents the surface magnetic characterization of two DAD samples with layer thickness of $15 \mathrm{~nm}$ and $20 \mathrm{~nm}$, respectively. Firstly, the hysteresis loops of DAD show single step magnetic behaviour in contrast to the multistep magnetic behaviour exhibited by the HAD with the same layer thickness, as show in figure $8 \mathrm{a}$, c. Also, the $H_{C}$ value of DAD is lower than the $H_{C}$ of the antidot with hexagonal ordering, but still larger than the corresponding value for a CTF with same thickness. This should be due to the domain wall pinning from disordered nanoholes. Secondly, the angular dependence of $H_{C}$ shows a random distribution as 
Influence of nanoholes array geometrical parameters

shown in figure $8 \mathrm{~b}$, because there is not any spatial ordering symmetry in the antidot structure, as shown in figure $1 \mathrm{a}$. However, the $H_{C}$ angular dependence for the DAD samples with $20 \mathrm{~nm}$ shows a two-fold order, such as in the case of the symmetry of CTF as show in figure $8 \mathrm{~d}$. This means that the induced shape anisotropy by disordered arrays of holes isn't big enough to overcome the intrinsic magnetic anisotropy of CTF.
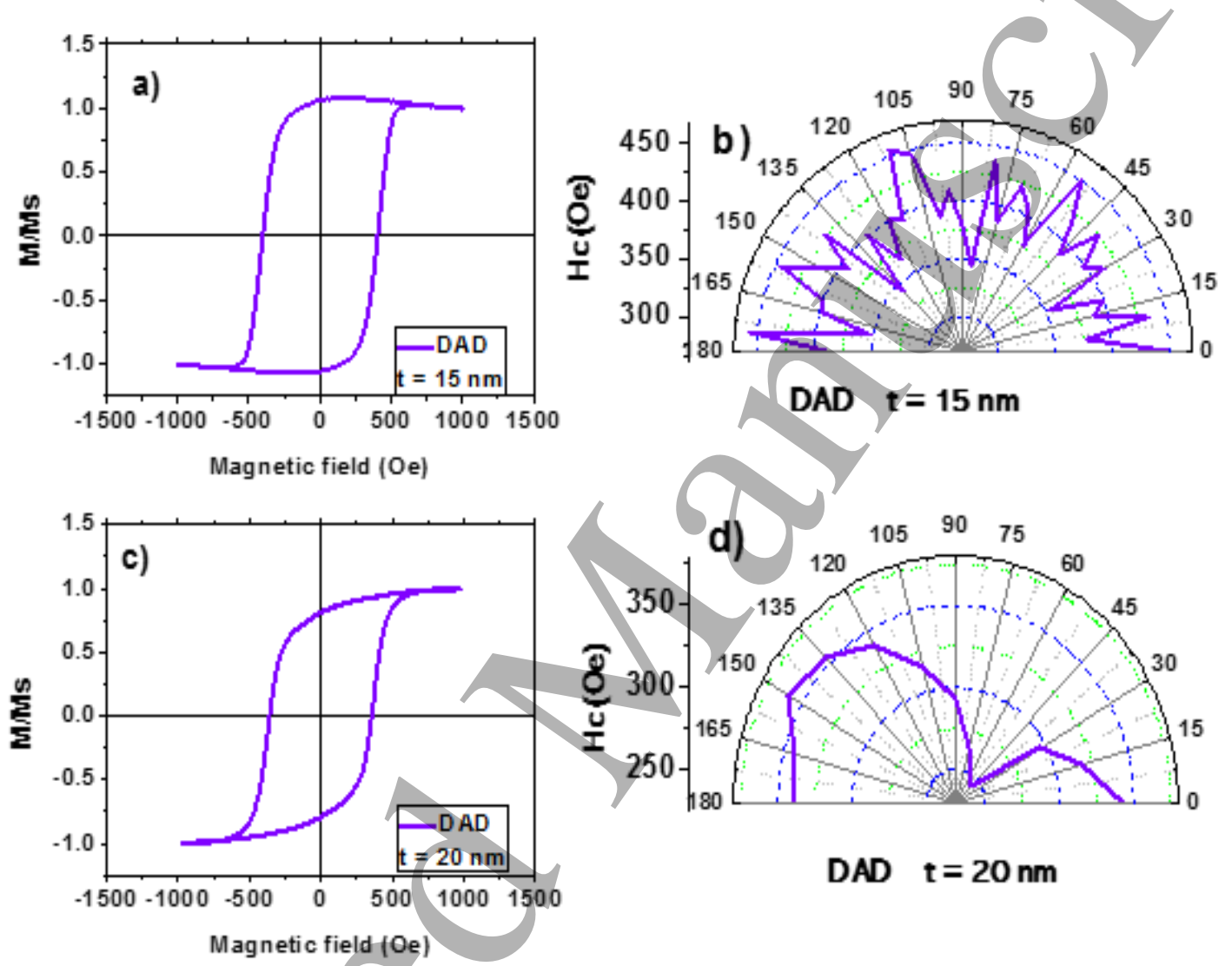

DAD $t=20 \mathrm{~nm}$

Figure 8: a) The in-plane hysteresis loops of Dy-Fe alloy disordered antidot array thin film with different thickness and related INP angular coercivity dependence for a) and b) $15 \mathrm{~nm}, \mathrm{c}$ ) and d) $20 \mathrm{~nm}$, respectively.

\subsection{Effective magnetic anisotropy for HAD and CTF}

An accurate calculation of the domain wall width for the antidot thin films can be derived from the local magnetic anisotropy. However, it is not possible to calculate the local stray fields that are responsible for the domain wall pinning in antidot thin films $[44,45]$. Therefore, a macroscopic model of the effective anisotropy would be used 
Influence of nanoholes array geometrical parameters

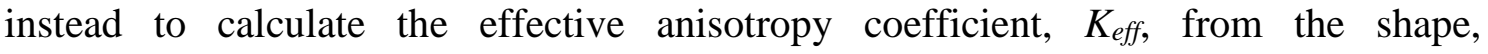
magnetostatic and demagnetizing anisotropies, because of the inclusion of the arrays of nano-holes into the CTF [44].Thereby, it is possible to obtain an approximation of the anisotropy of the antidot arrays by using a simple and straightforward method, and thus easily infer on the magnetic properties of the material. Experimentally, the $K_{\text {eff }}$ can be obtained from the in-plane and out-of-plane loops, by considering all the macroscopic effects, such as the defects and the dislocations between the hexagonal domains of antidots $[8,44]$

$$
\boldsymbol{K}_{e f f}=\int_{0-\mathrm{OOP}}^{\mathrm{M}_{\mathrm{s}}} \mathrm{HdM}-\int_{0-\mathrm{INP}}^{\mathrm{M}_{\mathrm{s}}} \mathrm{HdM}
$$

Where $\mathrm{M}$ is the magnetization, $\mathrm{M}_{\mathrm{S}}$ is the saturation magnetization and $\mathrm{H}$ is the applied magnetic field.

Figure $9(\mathrm{a}, \mathrm{b})$ represents the $K_{\text {eff }}$ and the effective anisotropy density, $K_{\text {eff }} \times t$, for both the Dy-Fe alloy HAD arrays and corresponding CTF with varying the layer thickness. For CTF samples both, the $K_{\text {eff }}$ and $K_{\text {eff }} \times t$ exhibit a nonlinear relationship with the layer thickness. The maximum value of $K_{\text {eff }}$ and $K_{\text {eff }} \times t$ occurred at the thickness value of $50 \mathrm{~nm}$, and then starts to decrease with increasing the layer thickness. This behavior can be attributed to a growth-induced modification of the microstructure of the amorphous films, which affects the short-range order [25]. As a result, a more collinear alignment of the distributed magnetic moments of Dy along the out-of-plane direction with film thickness is obtained [39]. It is worth noting that all CTF samples show a positive value of $K_{e f f}$ and $K_{\text {eff }} \times t$, i.e. the CTFs have an INP easy magnetization axis. 
Influence of nanoholes array geometrical parameters
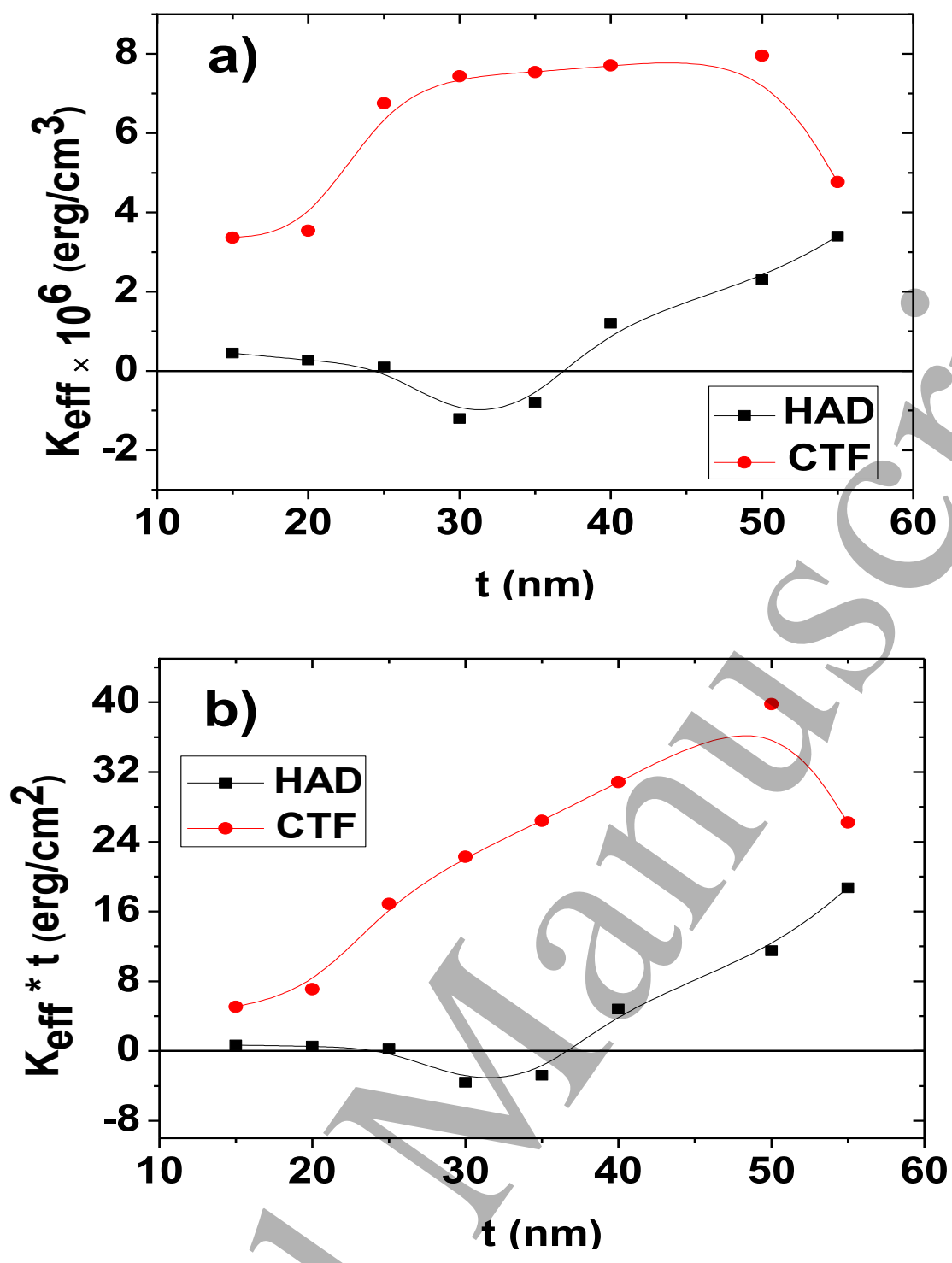

Figure 9: a) Effective anisotropy $K_{\text {eff }}$ and b) effective anisotropy density $K_{\text {eff }} \times t$ for Dy-Fe HAD and their corresponding CTF, plotted versus the film thickness. Negative values of $K_{\text {eff }}$ and $K_{\text {eff }} \times t$ indicate samples with perpendicular (OOP) anisotropy. The lines are guides to the eye.

The antidot samples display noticeable changes in the effective magnetic anisotropy. Firstly, $K_{\text {eff }}$ values sharply decrease for the HAD in comparison to the CTF due to the competition between the intrinsic anisotropy and the shape anisotropy of nanoholes and the contribution of the out-of-plane magnetic component to the magnetization reversal process [35-40].Therefore, it can be observed a magnetic anisotropy crossover from the in-plane to out-of-plane directions for antidot samples with layer thickness of $30 \mathrm{~nm}$ and $35 \mathrm{~nm}$ (negative values of $K_{\text {eff }}$ and $K_{\text {eff }} \times t$ ). It is worth to 
Influence of nanoholes array geometrical parameters

mention that the larger values of the effective perpendicular magnetic anisotropy energy density, $\left(K_{\text {eff }} \times t=3.8 \mathrm{erg} / \mathrm{cm}^{2}\right)$, which have been obtained for the material employed in this work make it as an excellent candidate for magneto-optic perpendicular recording patterned media based on template-assisted deposition techniques [8]. Finally, for the thicker HAD samples, the positive values obtained for both, $K_{\text {eff }}$ and $K_{\text {eff }} \times t$, closest to that of CTF, indicate an in-plane easy magnetization axis.

\subsection{Effect of hexagonal arrangement of Dy-Fe antidots on Curie temperature.}

The effects of thermal activation on the magnetic properties of the Dy-Fe antidot arrays and the continuous thin film, have been studied through the measurements of temperature dependence of the magnetization, $\mathrm{M}(\mathrm{T})$, in the temperature range between the room temperature, $\mathrm{RT}$, and $1000 \mathrm{~K}$.

Figure 10 shows the normalized magnetization, $\mathrm{M}(\mathrm{T}) / \mathrm{M}(\mathrm{RT})$, as a function of temperature of Dy-Fe CTF and corresponding antidot arrays film sample with layer thickness of $15 \mathrm{~nm}$ obtained by VSM technique. The magnetization curves for HAD and CTF have been obtained after subtracting from the measurements the signal of the corresponding substrates, glass in case of CTF and alumina for HAD. As show in figure 10, from $300 \mathrm{~K}$ to $550 \mathrm{~K}$ the magnetization behaviour for antidot arrays and CTF shows quite similar trend. An increase in the slope of magnetization curve for the CTF sample with temperature increasing can be observed comparing to HAD sample, ascribed to the existing hexagonal arrangement of antidots which may increase the thermal stability of hosting magnetic materials compare to $\mathrm{CTF}[46]$. A sharp drop in the magnetization curve of HAD sample has been observed around $785 \mathrm{~K}$ because of damage of the supporting alumina membrane when temperature raises. From $820 \mathrm{~K}$ to $860 \mathrm{~K}$ the magnetization curve for $\mathrm{HAD}$ and $\mathrm{CTF}$ samples reach the lowest value. Further increasing of 


\section{Influence of nanoholes array geometrical parameters}

temperature, leads to the HAD sample to show a strong paramagnetic phase due to the crystallization under the effect of high temperature annealing for $\mathrm{T}>860 \mathrm{~K}$.

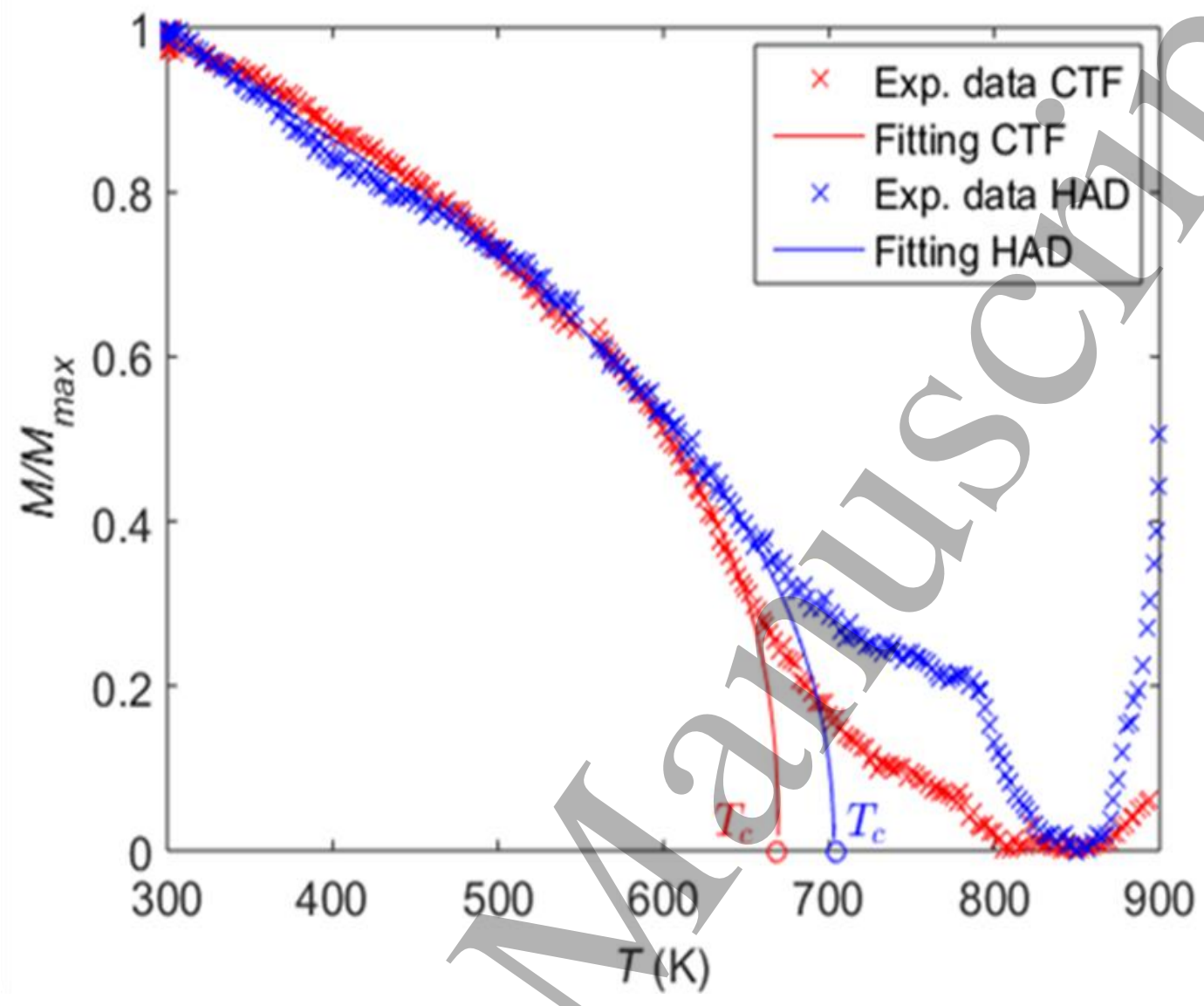

Figure 10: Temperature dependence of the magnetization in Dy-Fe alloys for both, CTF and HAD samples with layer thickness of $15 \mathrm{~nm}$, measured at constant applied field of $20 \mathrm{kOe}$ along the INP direction.

In order to obtain an accurate value of Curie temperature, $T_{C}$, a fitting of the magnetization curyes plotted in figure 10 was performed according to the law $[M(T, H)=$ $\left.M_{0}\left(\frac{T-T_{c}}{T_{c}}\right)^{\beta}\right][47]$. The $T_{C}$ increases from $669 \mathrm{~K}$ for CTF to $710 \mathrm{~K}$ in case of antidots array. This increase in the $T_{C}$ is attributed to the strong pinning effect $[48,49]$ induced by the nanoholes of the HAD sample. In addition, the hexagonal arrays of antidots exhibit high thermal stability due to three-dimensional magnetization profile where, the out-of-plane component plays a more important role due to the misaligned magnetic moments at the 


\section{Influence of nanoholes array geometrical parameters}

edge and at the inner wall of the nanoholes [46,50]. Meanwhile, the continuous thin film has only two-dimensional magnetization in-plane components, so the magnetic moments will need more thermal energy to overcome the ordered structure in the case of sample with HAD arrays.

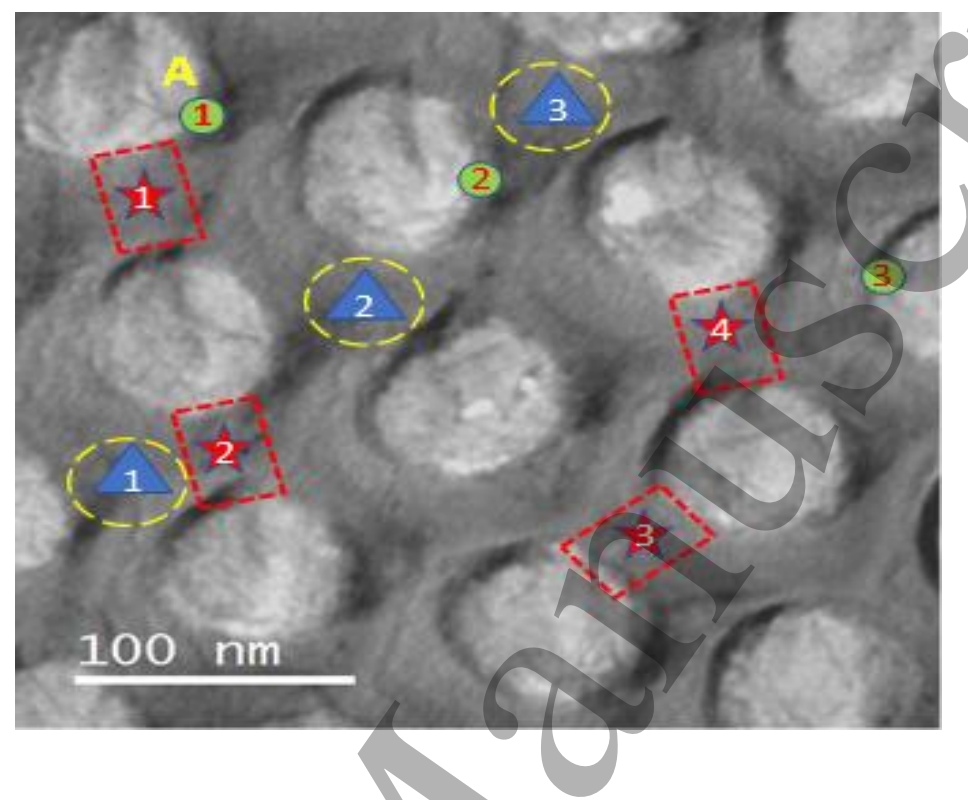

Figure 11: HR-TEM image for $\mathrm{Dy}_{13} \mathrm{Fe}_{87}$ antidot arrays shows the Dy and Fe elements distribution for different regions: 1) at the edge of holes (green circles from 1 to 3), 2) areas between two holes (red stars from 1 to 4), and the area between three holes (blue triangles from 1 to 3 ).

Figure 11 shows the HR-TEM image with elemental analysis for Fe and Dy at three different places. From the chemical analysis of the TEM images around the hexagonal unit cell of the Dy-Fe antidot arrays we find that composition of Dy-Fe alloys differs from place to place. Around the hole's edges, the analysis of the spectra of three regions shows $\mathrm{a} \mathrm{Dy}_{4} \mathrm{Fe}_{96}$ averaged composition, as summarized in Table 2. In contrast, for the area between two holes, W, which are marked as dotted red rectangles in Figure 11 , the chemical analysis of the four regions gives a $\mathrm{Dy}_{28} \mathrm{Fe}_{72}$ averaged composition. Finally, for the wide area confined between three holes, the Dy-Fe alloy exhibits an averaged composition of $\mathrm{Dy}_{13} \mathrm{Fe}_{87}$, which is very close to the continuous thin film values. Thus, the existence of iron rich areas with $\mathrm{Dy}_{4} \mathrm{Fe}_{96}$ alloy composition in case of hexagonal 


\section{Influence of nanoholes array geometrical parameters}

antidot arrays may lead to the observed increase in $T_{C}$ for the antidots sample as compared to the CTF, as plotted in Figure10.

Table 2: Atomic percentage of Fe and Dy elements measured at different zones of the antidot sample.

\begin{tabular}{|l|l|l|l|l|l|l|}
\hline Region & \multicolumn{2}{|c|}{$\begin{array}{c}\text { Area around the holes } \\
\text { (green dots) }\end{array}$} & $\begin{array}{c}\text { Area between two holes } \\
\text { (red stars) }\end{array}$ & $\begin{array}{c}\text { Area between three holes } \\
\text { (blue triangles) }\end{array}$ \\
\hline Spectrum & Fe (at. \%) & Dy (at. \%) & Fe (at. \%) & Dy (at. \%) & Fe (at. \%) & Dy (at. \%) \\
\hline 1 & 95.0 & 5.0 & 76.0 & 24.0 & 89.0 & 11.0 \\
\hline 2 & 97.0 & 3.0 & 77.0 & 23.0 & 84.8 & 15.2 \\
\hline 3 & 96.5 & 3.5 & 71.0 & 29.0 & 87.5 & 12.5 \\
\hline 4 & $\mathbf{9 6 . 2}$ & $\mathbf{3 . 8}$ & $\mathbf{7 2 . 3}$ & $\mathbf{2 7 . 7}$ & $\mathbf{8 7 . 1}$ & $\mathbf{1 2 . 9}$ \\
\hline Average & $\mathbf{9 6 . 0}$ & & & & & \\
\hline
\end{tabular}

The magnetic behaviour of Dy-Fe alloy HAD and corresponding CTF with different layer thickness have been investigated. The dependence of the magnetization reversal mechanism on their lattice parameters have been analysed through micro-andmacroscopic angular dependence of the in-plane coercivity. The microscopic local measurements of the in-plane magnetic anisotropy for antidot arrays samples show a noticeable change with increasing the layer thickness, exhibiting a magnetization crossover at the thickness value of $25 \mathrm{~nm}$. For thinner antidot sample, $W<d$, tri-axial magnetic anisotropy has been detected. Meanwhile, for thicker antidot arrays samples, $W$ $>d$, an uniaxial magnetic anisotropy has been observed. A strong correlation between the edge-to-edge distance and the local magnetic anisotropy of the Dy-Fe alloyed antidot arrays samples has been found. Also, the competition between the intrinsic magnetic anisotropy of CTF and the magnetic shape anisotropy plays the main role on the effective 
Influence of nanoholes array geometrical parameters

magnetic anisotropy behaviour of the antidot arrays samples. The effective magnetic anisotropy shows a crossover from the in-plane easy magnetization axis to out-of-plane easy axis by increasing the layer thickness at the critical value of $25 \mathrm{~nm}$. Furthermore, the thermal magnetization behavior for antidot arrays shows an increase in the Curie temperature point comparing to that of the CTF due to the high thermal stability induced by the local hexagonal symmetry of nanoholes arrangement for the HAD sample. Finally, the strong dependence of magnetic properties of Dy-Fe alloys antidot arrays on the layer thickness and temperature may advance 2D magnetic logic to the third dimension not only at room temperature, but also at high temperature. In addition, these observations can be of high interest for the development of novel magnetic sensors with high uniaxial magnetic anisotropy and for thermo-magnetic recording patterned media based on template-assisted deposition techniques.

\section{Acknowledgements}

This work has been financially supported under Spanish MINECO research Project No. MAT2016-76824-C3-3-R. Nanoporous Membranes Laboratory and research facilities from SCTs of Universidad de Oviedo are also recognized. Authors wish to acknowledge Dr. M. Ipatov the help provided with magnetic measurements, from SGIker Medidas Magnéticas unit, from University of Basque Country (UPV/EHU). 
Influence of nanoholes array geometrical parameters

\section{References:}

[1] Lenk B, Ulrichs H, Garbs F and Münzenberg M 2011 The building blocks of magnonics Phys. Rep. 507 107-36

[2] Yu H, Duerr G, Huber R, Bahr M, Schwarze T, Brandl F and Grundler D 2013 Omnidirectional spin-wave nanograting coupler Nat. Commun. 42702

[3] Jalil M B A 2003 Bit isolation in periodic antidot arrays using transverse applied fields J. Appl. Phys. 93 7053-5

[4] Torres L, Lopez-Diaz L and Iñiguez J 1998 Micromagnetic tailoring of periodic antidot permalloy arrays for high density storage Appl. Phys. Lett. 73 3766-8

[5] Wang R F, Nisoli C, Freitas R S, Li J, McConville W, Cooley B J, Lund M S, Samarth N, Leighton C, Crespi V H and Schiffer P 2006 Artificial "spin ice" in a geometrically frustrated lattice of nanoscale ferromagnetic islands Nature 439 303-306

[6] Belotelov V I, Kreilkamp L E, Akimov I A, Kalish A N, Bykov D A, Kasture S, Yallapragada V J, Gopal A V, Grishin A M, Khartsev S I, Nur-E-Alam M, Vasiliev M, Doskolovich L L, Yakovlev D R, Alameh K, Zvezdin A K and Bayer M 2013 Plasmon-mediated magneto-optical transparency Nat. Commun. 42128

[7] Melkov G A, Vasyuchka V I, Chumak A V., Tiberkevich V S and Slavin A N 2006 Wave front reversal of nonreciprocal surface dipolar spin waves J. Appl. Phys. 99 8-11

[8] Salaheldeen M, Vega V, Ibabe A, Jaafar M, Asenjo A, Fernandez A and Prida V M 2018 Tailoring of Perpendicular Magnetic Anisotropy in Dy13Fe87 Thin Films with Hexagonal Antidot Lattice Nanostructure Nanomaterials 8227

[9] Metaxas P J, Sushruth M, Begley R A, Ding J, Woodward R C, Maksymov I S, Albert M, Wang W, Fangohr H, Adeyeye A O and Kostylev M 2015 Sensing magnetic nanoparticles using nano-confined ferromagnetic resonances in a magnonic crystal Appl. Phys. Lett. 106232406

[10] Yu C T, Jiang H, Shen L, Flanders P J and Mankey G J 2000 The magnetic anisotropy and domain structure of permalloy antidot arrays J. Appl. Phys. 87 $6322-4$

[11] García-Snchez F, Paz E, Pigazo F, Chubykalo-Fesenko O, Palomares F J, González J M, Cebollada F, Bartolomé J and García L M 2008 Coercivity mechanisms in lithographed antidot arrays Epl $\mathbf{8 4}$

[12] S Michea, JLPalma, RLav'1n , J Briones, J Escrig J C D and R L R-S 2014 Tailoring the magnetic properties of cobalt antidot arrays by varying the pore size and degree of disorder J. Phys. D. Appl. Phys. 47

[13] Prida V M, Salaheldeen M, Pfitzer G, Hidalgo A, Vega V, González S, Teixeira J M, Fernández A and Hernando B 2017 Template assisted deposition of ferromagnetic nanostructures: From antidot thin films to multisegmented nanowires Acta Phys. Pol. A 131 822-7

[14] De A, Mondal S, Sahoo S, Barman S, Otani Y, Mitra R K and Barman A 2018 Field-controlled ultrafast magnetization dynamics in two-dimensional nanoscale 
Influence of nanoholes array geometrical parameters

ferromagnetic antidot arrays Beilstein J. Nanotechnol. 9 1123-1134

[15] Wang C C, Adeyeye A O and Singh N 2006 Magnetic antidot nanostructures: Effect of lattice geometry Nanotechnology 17 1629-36

[16] Merazzo K J, Del Real R P, Asenjo A and Vzquez M 2011 Dependence of magnetization process on thickness of Permalloy antidot arrays J. Appl. Phys. 109 $07 \mathrm{~B} 906$

[17] Choudhury S, Majumder S, Barman S, Otani Y and Barman A 2018 Active Control of Mode Crossover and Mode Hopping of Spin Waves in a Ferromagnetic Antidot Lattice Phys. Rev. Appl. 10064044

[18] Garad H, Usmani S, Barral D, David P, Cagnon L, Testemale D, Mannix D, Fettar F, Proux O, Rosa A, Mathon O and Pascarelli S 2018 Influence of the pore diameter in $\mathrm{Cu} / \mathrm{Co} / \mathrm{Cu}$ antidots: A XANES study Phys. Rev. Mater. 2066001

[19] Radu F, Abrudan R, Radu I, Schmitz D and Zabel H 2012 Perpendicular exchange bias in ferrimagnetic spin valves Nat. Commun. 3 715-7

[20] I. Radu, K.Vahaplar, C. Stamm, T.Kachel, N. Pontius, H. A. Dürr, T. A. Ostler, J. Barker, R. F.L.Evans, R.W.Chantrell, A. Tsukamoto, A.Itoh, A. Kirilyuk T R and A V K 2011 Transient ferromagnetic-like state mediating ultrafast reversal of antiferromagnetically coupled spins Nature 472 205-208

[21] Hassdenteufel A, Hebler B, Schubert C, Liebig A, Teich M, Helm M, Aeschlimann M, Albrecht M and Bratschitsch R 2013 Thermally Assisted AllOptical Helicity Dependent Magnetic Switching in Amorphous Fe $100-\mathrm{x}$ Tb x Alloy Films Adv. Mater. 25 3122-8

[22] K. Fleury-Frenette, J. Delwiche, F. Grandjean, D. Vandormael and G J L 2001 Magnetic and conversion electron Mössbauer spectral study of amorphous thin films of Dy x Fe 100 - x and Dy 20 Fe 80 - y Co y J. Appl. Phys. 90 1934-40

[23] Tang M H, Zhang Z, Tian S Y, Wang J, Ma B and Jin Q Y 2015 Interfacial exchange coupling and magnetization reversal in perpendicular $[\mathrm{Co} / \mathrm{Ni}] \mathrm{N} / \mathrm{TbCo}$ composite structures Sci. Rep. 51-7

[24] Fust S, Mukherjee S, Paul N, Stahn J, Kreuzpaintner W, Böni P and Paul A 2016 Realizing topological stability of magnetic helices in exchange-coupled multilayers for all-spin-based system Sci. Rep. 6 1-14

[25] Hebler B, Hassdenteufel A, Reinhardt P, Karl H and Albrecht M 2016 Ferrimagnetic Tb-Fe Alloy Thin Films: Composition and Thickness Dependence of Magnetic Properties and All-Optical Switching Front. Mater. 3 1-8

[26] Hansen P, Klahn S, Clausen C, Much G and Witter K 1991 Magnetic and magneto-optical properties of rare-earth transition-metal alloys containing Dy, Ho, Fe, Co J. Appl. Phys. 69 3194-207

[27] Liao J, He H, Zhang Z, Ma B and Jin Q Y 2011 Enhanced difference in switching fields for perpendicular magnetic spin valves with a composite [Co/Ni]NTbCo reference layer J. Appl. Phys. 1093907

[28] Kulesh N A, Vázquez M, Lepalovskij V N and Vas'Kovskiy V O 2018 Antidot patterned single and bilayer thin films based on ferrimagnetic Tb-Co alloy with 
Influence of nanoholes array geometrical parameters

[31] López Antón R, Vega V, Prida V M, Fernández A, Pirota K R and Vázquez M 2009 Magnetic Properties of Hexagonally Ordered Arrays of Fe Antidots by Vacuum Thermal Evaporation on Nanoporous Alumina Templates Solid State Phenom. 152-153 273-6

[32] Masuda H and Fukuda K 1995 Ordered Metal Nanohole Arrays Made by a TwoStep Replication of Honeycomb Structures of Anodic Alumina Science (80-. ). 268 1466-8

[33] Béron F, Pirota K R, Vega V, Prida V M, Fernández A, Hernando B and Knobel M 2011 An effective method to probe local magnetostatic properties in a nanometric FePd antidot array New J. Phys. 13 3035-51

[34] Svalov A V., Fernandez A, Vas'kovskiy V O, Kurlyandskaya G V., Barandiarán J M, Anton R L and Tejedor M 2006 MOKE study of $\mathrm{Co} / \mathrm{Ti} /(\mathrm{Gd}-\mathrm{Co})$ multilayers near the magnetic compensation state J. Alloys Compd. 419 25-31

[35] Salaheldeen M, Méndez M, Vega V, Fernández A and Prida V M 2019 Tuning Nanohole Sizes in Ni Hexagonal Antidot Arrays: Large Perpendicular Magnetic Anisotropy for Spintronic Applications ACS Appl. Nano Mater. 2 1866-1875

[36] Wang C C, Adeyeye A O and Singh N 2006 Magnetic antidot nanostructures: Effect of lattice geometry Nanotechnology 171629

[37] Leamy H J and Dirks A G 1979 Microstructure and magnetism in amorphous rareearth-transition-metal thín films. II. Magnetic anisotropy J. Appl. Phys. 502871

[38] Leamy H J and Dirks A G 1978 Microstructure and magnetism in amorphous rareearth-transition-metal thin films. I. Microstructure J. Appl. Phys. 493034

[39] R. Carey, L. Dieu and D. M. Newman 1997 Thickness variation of the magnetic anisotropy in DyFe thin films using magneto-optical techniques IEEE Trans. Magn. 33 3922-4

[40] Merazzo K J, Castán-Guerrero C, Herrero-Albillos J, Kronast F, Bartolomé F, Bartolomé J, Sesé J, Del Real R P, García L M and Vázquez M 2012 X-ray photoemission electron microscopy studies of local magnetization in Py antidot array thin films Phys. Rev. B - Condens. Matter Mater. Phys. 85184427

[41] Jang Y H and Cho J H 2014 Morphology-dependent multi-step ferromagnetic reversal processes in Co thin films on crescent-shaped antidot arrays J. Appl. Phys. 115063903

[42] Hu X K, Sievers S, Janke V and Schumacher H W 2011 Classification of super domains and super domain walls in permalloy antidot lattices Phys. Rev. B 84 024404 
Influence of nanoholes array geometrical parameters

[43] Haering F, Wiedwald U, Nothelfer S, Koslowski B, Ziemann P, Lechner L, Wallucks A, Lebecki K, Nowak U, Gräfe J, Goering E and Schütz G 2013 Switching modes in easy and hard axis magnetic reversal in a self-assembled antidot array Nanotechnology $\mathbf{2 4}$

[44] Navas D, Hernández-Vélez M, Vázquez M, Lee W and Nielsch K 2007 Ordered $\mathrm{Ni}$ nanohole arrays with engineered geometrical aspects and magnetic anisotropy Appl. Phys. Lett. 90192501

[45] Merazzo K J, Leitao D C, Jiménez E, Araujo J P, Camarero J, Del Real R P, Asenjo A and Vázquez M 2011 Geometry-dependent magnetization reversal mechanism in ordered Py antidot arrays J. Phys. D. Appl. Phys. 44 5001-10

[46] Gong W J, Yu W J, Liu W, Guo S, Ma S, Feng J N, Li B and Zhang Z D 2012 Exchange bias and its thermal stability in ferromagnetic/antiferromagnetic antidot arrays Appl. Phys. Lett. 1012407

[47] Caballero-Flores R, Bingham N S, Phan M H, Torija M A, Leighton C, Franco V, Conde A, Phan T L, Yu S C and Srikanth H 2014 Magnetocaloric effect and critical behavior in Pr0.5Sr 0.5MnO3: An analysis of the validity of the Maxwell relation and the nature of the phase transitions J. Phys. Condens. Matter 26286001

[48] Punz D, Lee J, Fuger M, Fidler J, Schrefl T and Suess D 2010 Theory and micromagnetics of pinning mechanism at cylindrical defects in perpendicular magnetic films J. Appl. Phys. 107113926

[49] Tripathy D and Adeyeye A O 2011 Perpendicular anisotropy and out-of-plane exchange bias in nanoscale antidot arrays New J. Phys. 13023035

[50] Fettar F, Cagnon L and Rougemaille N 2010 Three-dimensional magnetization profile and multiaxes exchange bias in Co antidot arrays Appl. Phys. Lett. 97 200811 